ISSN: 0514-7336

DOI: https://doi.org/10.14201/zephyrus201678195201

\title{
EVALUACIÓN DE LAS MANIFESTACIONES GRÁFICAS PARIETALES DE LA CUEVA DEL FORCÓN (A FUEBA, HUESCA): NUEVAS PERSPECTIVAS SOBRE EL ARTE PALEOLÍTICO EN LA VERTIENTE SUR DEL PIRINEO CENTRAL
}

\section{Evaluation of the parietal graphic motifs from El Forcón Cave (A Fueba, Huesca): New Perspectives on the Palaeolithic Art in the Southern slope of the Central Pyrenees}

Aitor Ruiz Redondo*, Ignacio Clemente Conte**, Javier Rey Lanaspa***, Ermengol Gassiot Ballbék*** y Mikel EtXebarría CaSAs ${ }^{* * * * *}$

* pACEA (UMR5199)-Univ. de Bordeaux. Bât. B18. Allée Geoffroy Saint-Hilaire. 33615 Pessac CEDEX (France). Correo-e:aruizredondo@gmail.com

** Intitució Milá i Fontanals-CSIC. C/ Egipcíaques, 15.08001 Barcelona. Correo-e: ignacio@imf.csic.es

*** Dpto. de Educación, Cultura y Deporte. Gobierno de Aragón

**** Dpto. de Prehistoria. Edificio B. Facultad de Filosofía y Letras-UAB. 08193 Bellaterra (Barcelona). Correo-e: ermengol.gassiot@uab.cat

***** GEONATURA-Guaso. Correo-e: geonatura@hotmail.com.

Recepción: 1/07/2016; Revisión: 6/07/2016; Aceptación: 2/09/2016

Resumen: En 1976, un equipo dirigido desde el Museo de Huesca realizó una serie de trabajos en la Cueva del Forcón (San Juan de Toledo, A Fueba, Huesca). Además de la recuperación de diversos materiales e instrumentos prehistóricos en un contexto totalmente alterado, se constató la existencia de grabados parietales de origen antrópico. Tras el descubrimiento del conjunto paleolítico de la Fuente del Trucho, también en la vertiente sur del Pirineo Central, y debido a la similitud formal de los grabados del Forcón con los de otros conjuntos franco-cantábricos, se propuso una cronología paleolítica para el 'arte parietal' de este yacimiento. Desde entonces, la literatura científica lo ha incluido en el inventario de conjuntos parietales paleolíticos. Recientemente, emprendimos un nuevo estudio del dispositivo gráfico -sin revisar desde su primera publicación-, para evaluar su potencial y los argumentos que ofrece para establecer una cronología. En este artículo discutimos las evidencias encontradas y presentamos las conclusiones de este estudio. La principal es que los principales argumentos hallados no avalan una cronología paleolítica -ni incluso prehistórica- de estos motivos.

Palabras clave: Arte parietal; norte peninsular; grabados; trazos digitales; cronología.

Aвstract: In 1976, a team led from the Museum of Huesca conducted a series of archaeological works in El Forcón Cave (San Juan de Toledo, A Fueba, Huesca). In addition to the recovery of several materials and prehistoric tools in a completely disturbed context, it was discovered the existence of parietal anthropic engravings. After the discovery of the Palaeolithic parietal site of Fuente del Trucho, also on the southern 
slope of the Central Pyrenees, and joined to the formal similarity of the El Forcón engravings with other Franco-Cantabrian ensembles, a Palaeolithic chronology was proposed for the 'parietal art' of this site. Since then, the scientific literature has included this ensemble in the inventory of cave art. Recently, we undertook a study of the graphical device -unrevised since its first publication-, to assess its potential and the arguments to establish a chronology. In this paper we discuss the evidence found and present the conclusions of the study. The most relevant is that the arguments do not support a Palaeolithic -or even a Prehistoric- chronology for the parietal motifs.

Key words: Rock Art; Northern peninsular; engravings; finger flutings; chronology.

\section{Introducción ${ }^{1}$}

La Cueva del Forcón se abre en los escarpados acantilados calizos de la Sierra Ferrera, entre los valles del Cinca y el Ésera. Se ubica en la localidad de San Juan de Toledo, en el término de A Fueba (Huesca), en las coordenadas UTM 270707x; 4705873y (datum ETRS89) y a una altitud de $1383 \mathrm{msnm}$ (Fig. 1). La aproximación a la cueva es costosa, a casi $3 \mathrm{~h}$ de ascenso campo a través desde el camino del núcleo habitado más cercano, San Juan de Toledo. La cueva presenta un acceso complicado, en que se hace necesario el uso de equipo y técnicas de escalada para salvar la pared vertical de $7 \mathrm{~m}$ que

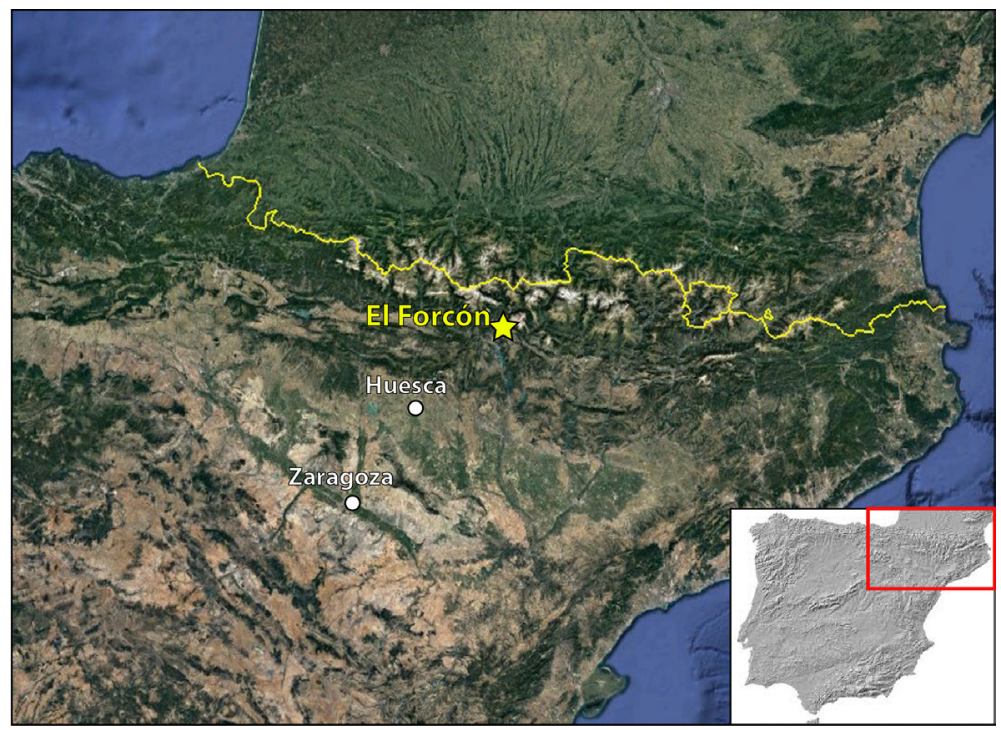

Fig. 1. Mapa con la ubicación de la Cueva del Forcón. da acceso a la repisa en que se abre la boca inferior de la cavidad. Cuenta asimismo con otra entrada en un nivel superior donde se localizó una pieza de bronce romana (Marco Simón, 1985), y que está comunicada con la primera a través de una chimenea vertical de unos $5 \mathrm{~m}$ que da acceso a la sala vestibular. Desde ésta se accede a una galería baja, de entre 3 y $7 \mathrm{~m}$ de anchura, que se desarrolla linealmente en dirección $\mathrm{E}$ durante unos $100 \mathrm{~m}$. Al final de ésta, otra galería parte entonces en dirección $\mathrm{N}$ y de forma ascendente a lo largo de unos $40 \mathrm{~m}$, donde termina el desarrollo transitable de la cavidad.

La roca madre aflora en gran parte de su desarrollo, sobre todo en la primera mitad. Antes del final

1 Este estudio ha recibido apoyo financiero del Estado francés, a través de un contrato postdoctoral, para el primer firmante (ARR), en el marco del programa Investments for the future IdEx Bordeaux (ANR-10-IDEX-03-02).

(C) Universidad de Salamanca

de la primera galería se localizan unos depósitos de arena fluvial, de granulometría muy fina. El paso por esta superficie es obligado para acceder a la parte más interna de la cueva y, sin embargo, las arenas aparecieron inalteradas desde largo tiempo, con las marcas superficiales del goteo del techo, dando cuenta del reducido tránsito por ella en las últimas décadas, lo cual, sumado a su difícil acceso, podría avalar la preservación de motivos parietales de época prehistórica.

La cueva fue descubierta en los ańos 50, cuando algunos vecinos dieron cuenta de la existencia de restos humanos en la superficie del vestíbulo de la cavidad. Estos restos y su sedimento fueron extraídos por miembros de la Guardia Civil, desconociéndose en la actualidad su cantidad, calidad y paradero (Baldellou, 1985: 153).

Dos décadas más tarde, se llevó a cabo una serie de trabajos propiamente arqueológicos para evaluar 
el potencial del yacimiento. En 1976, se realizaron varias catas en el vestíbulo y en la zona de los motivos parietales (Baldellou, 1985: 153). En las del vestíbulo se constata una estratigrafía de poca potencia y totalmente revuelta por las intervenciones de furtivos, donde se recuperaron una serie de materiales de diverso carácter: a) fragmentos cerámicos (los más abundantes, algunos de ellos decorados); b) muy escasos restos líticos (10 piezas no retocadas, dos piezas de hoz y dos láminas con retoque plano; así como un fragmento de filo de hacha pulimentada de basalto); c) alguna pieza de industria ósea (dos punzones y un fragmento de otro); d) objetos de adorno (39 cuentas discoidales, la mayoría realizadas sobre concha, una cuenta de Dentalium y un fragmento de otra), y e) restos óseos, tanto humanos como animales, en su mayoría de especies domésticas. Las catas de la zona "decorada" resultaron, sin embargo, estériles. El autor señala que las producciones cerámicas son las más determinantes para apuntar una cronología del depósito, situando -al menos- dos momentos, uno en una fase avanzada del Neolítico Final y otro en el Calcolítico (Baldellou, 1985: 160-161), por comparación con las cerámicas del muy próximo yacimiento de la Espluga de la Puyascada (Baldellou, 1976, 1987).

Por otro lado, se recuperó una plaqueta de bronce romana con grabados zoomorfos -un caballo y varios jabalíes- en la segunda entrada de la cueva -la superior-, fuera de contexto y que parece no tener relación ni espacial ni cronológica con el resto de materiales (Marco Simón, 1985). Por último, se realizó un somero estudio de las manifestaciones parietales del fondo de la cavidad (Casado López, 1985). Éstas consisten en una serie de grabados digitales, de los denominados macarroni o finger flutings (Sharpe y Van Gelder, 2006). En esta publicación se describen los motivos, se indica la ausencia de representaciones figurativas -a excepción de un posible équido, que se presenta con muchas reservas- y propone una cronología paleolítica para los mismos, basándose en comparaciones con conjuntos franco-cantábricos y en la proximidad -relativa- de la estación paleolítica de la Fuente del Trucho (Casado López, 1985: 189). Esta asignación cronológica se ha mantenido hasta

(C) Universidad de Salamanca la actualidad (e. g. Baldellou, 1990; Beltrán, 1990, 1997), si bien algún autor ha destacado la debilidad de estos argumentos (Utrilla, 2000).

\section{Objetivos y metodología}

Tras el descubrimiento de algunos motivos parietales similares a los descritos en la cueva de Coro Trasito (Ruiz-Redondo, 2015) se planteó la necesidad de reevaluar el dispositivo gráfico de la próxima Cueva de El Forcón con el fin de intentar contextualizar los primeros.

La prospección parietal y evaluación de las evidencias gráficas del Forcón (A Fueba, Huesca) fue realizada, por los firmantes, con el fin último de revisar los motivos parietales publicados en los años 80. Los objetivos de la actuación fueron:

1. Revisar la naturaleza de los motivos publicados.

2. Documentar la posible presencia de nuevas manifestaciones gráficas.

3. Comprobar la existencia de sedimentos susceptibles de albergar restos de carácter arqueológico.

4. Evaluar la antigüedad y posible adscripción cronológica del dispositivo gráfico de la cavidad.

Para la ejecución del trabajo de campo se contó con 4 linternas frontales Petzl ${ }^{\circ}$ Ultra Vario de 520 lúmenes y dos focos autónomos Workstar ${ }^{\circledR}$ Nightsearcher de 2200 w/1000 lúmenes. El trabajo se desarrolló mediante la observación directa y con ayuda de una lupa portátil de hasta 15x. Las fotografías se tomaron con una cámara réflex Sony ${ }^{\oplus} \alpha-230$ con un objetivo de 18-55 $\mathrm{mm}$ y sin ningún tipo de flashes externos. La evaluación de las evidencias se realizó sin ningún tipo de intervención directa sobre las mismas y, por lo tanto, sin alterar su estado de conservación.

\section{Descripción de los motivos parietales}

El conjunto de evidencias parietales se localiza efectivamente en la galería terminal de la cavidad. Estas son de varios tipos:

1. Trazos digitales sobre la arcilla: ocupan gran parte de la superficie del techo de los últimos

Zephyrus, LXXVIII, julio-diciembre 2016, 195-201 
$10 \mathrm{~m}$ de desarrollo de la galería terminal. Se trata en su mayoría de trazos digitales -otros realizados con algún instrumento sobre la arcilla fresca-, que se mezclan con marcas de zarpazos de diferentes tipos de mamíferos. Aparecen normalmente en forma de series de dos o tres líneas paralelas y de desarrollo rectilíneo que se entrecruzan entre sí, en ocasiones formando paneles de cierta complejidad (Fig. 2, n. ${ }^{0}$ 1). Ninguno conforma una figura ni signo convencional reconocible. La superficie total cubierta por estos motivos puede superar los $10 \mathrm{~m}^{2}$, repartidos en una decena de 'paneles' o acumulaciones de éstos.

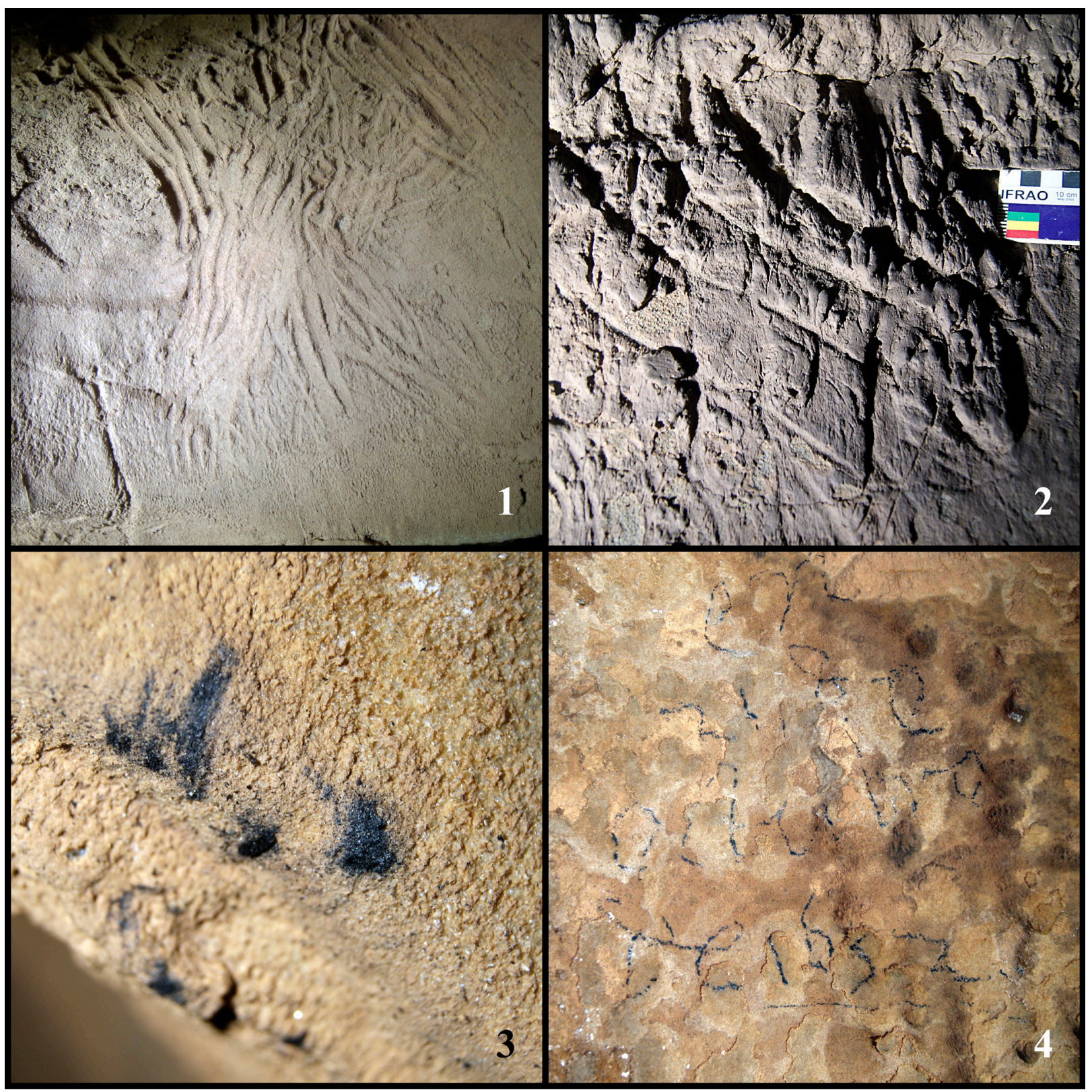

Fig. 2. Distintos tipos de motivos parietales en la Cueva del Forcón: 1) trazos digitales sobre arcilla; 2) zarpazos de origen animal; 3) trazos negros de carbón; 4) inscripciones de época histórica. 
2. Zarpazos de origen animal: se documentan mezclados con los trazos digitales, en ocasiones infrapuestos o superpuestos a éstos. Son de diverso carácter y origen; los más abundantes proceden de los murciélagos que aún habitan la cueva, pero también se registraron algunos de carnívoros de tamaño pequeño/medio -¿̨mustélido? ¿zorro?- e incluso de oso (Fig. 2, n. ${ }^{\circ}$ 2).

3. Trazos negros de carbón: aparecen en diferentes zonas, producto del contacto de alguna antorcha contra la superficie parietal. Tienen un aspecto reciente, incluso conservando alguno pequeños fragmentos de carbón adheridos a la pared (Fig. 2, n. ${ }^{\circ}$ 3).

4. Inscripciones de época histórica: no son abundantes en comparación con otras cuevas peninsulares, probablemente debido a su aislada situación y a la dificultad de su acceso. En el vestíbulo y la primera galería sólo se encontraron tres, probablemente todas ellas de la segunda mitad del s. Xx. En la galería final, en la zona de los trazos digitales, hay otras dos, que presentan cierto interés. La primera está grabada y aparece la fecha de 1936. La segunda se ha pintado con carbón en negro, con letra caligráfica y se puede leer: "En 21 de octubre de 1652" (Fig. 2, n. ${ }^{\circ} 4$ ).

\section{Discusión y evaluación}

A continuación se evalúan los motivos gráficos parietales a partir de las evidencias recopiladas durante el trabajo de campo.

En primer lugar resulta evidente el origen antrópico de los trazos digitales y otras marcas dejadas mediante el uso de algún instrumento. Sin embargo, la adscripción cronológica al Paleolítico Superior (e incluso a época prehistórica más reciente) nos parece más discutible. Existen una serie de argumentos que precisamente apuntan a un origen más reciente para la realización de esas marcas:

1. Morfología/estilo: no se observa ninguna figura reconocible. Los motivos se reducen a series rectilíneas simples, normalmente no mayores de 8-10 cm. Los macarroni o finger flutings de cronología paleolítica suelen presentar inflexiones y cambios de dirección en el trazo (Sharpe y Van Gelder, 2006). Por la acumulación de distintas series realizadas con varios dedos, da la impresión de que en algunos paneles pueden documentarse gestos más complejos, pero descomponiendo los trazos se puede comprobar que todos ellos son líneas simples y más o menos rectas (Fig. 3).

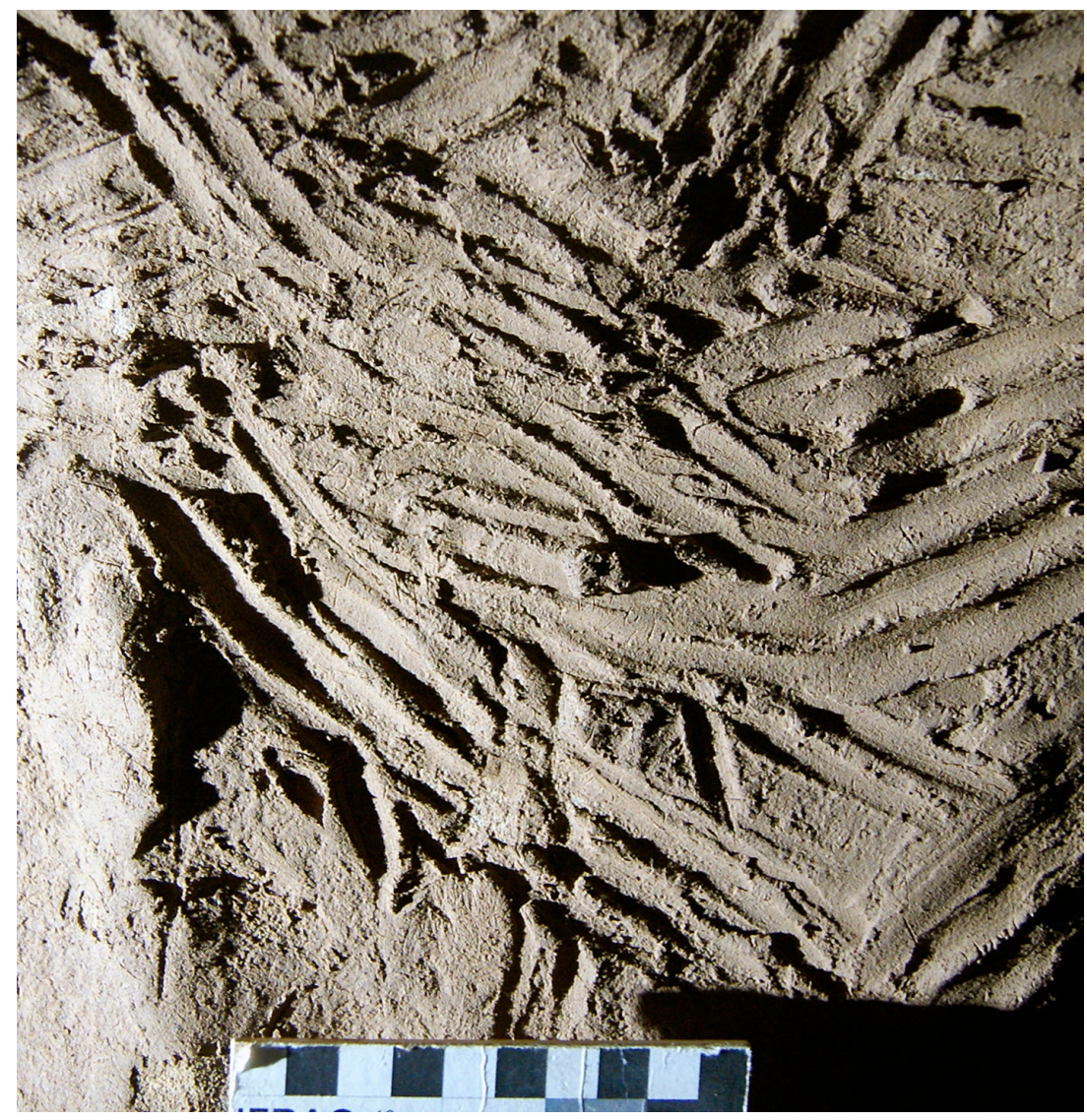

FIG. 3. Panel de trazos digitales en el que se puede apreciar la ausencia de pátina y erosión y la presencia de rebabas. 


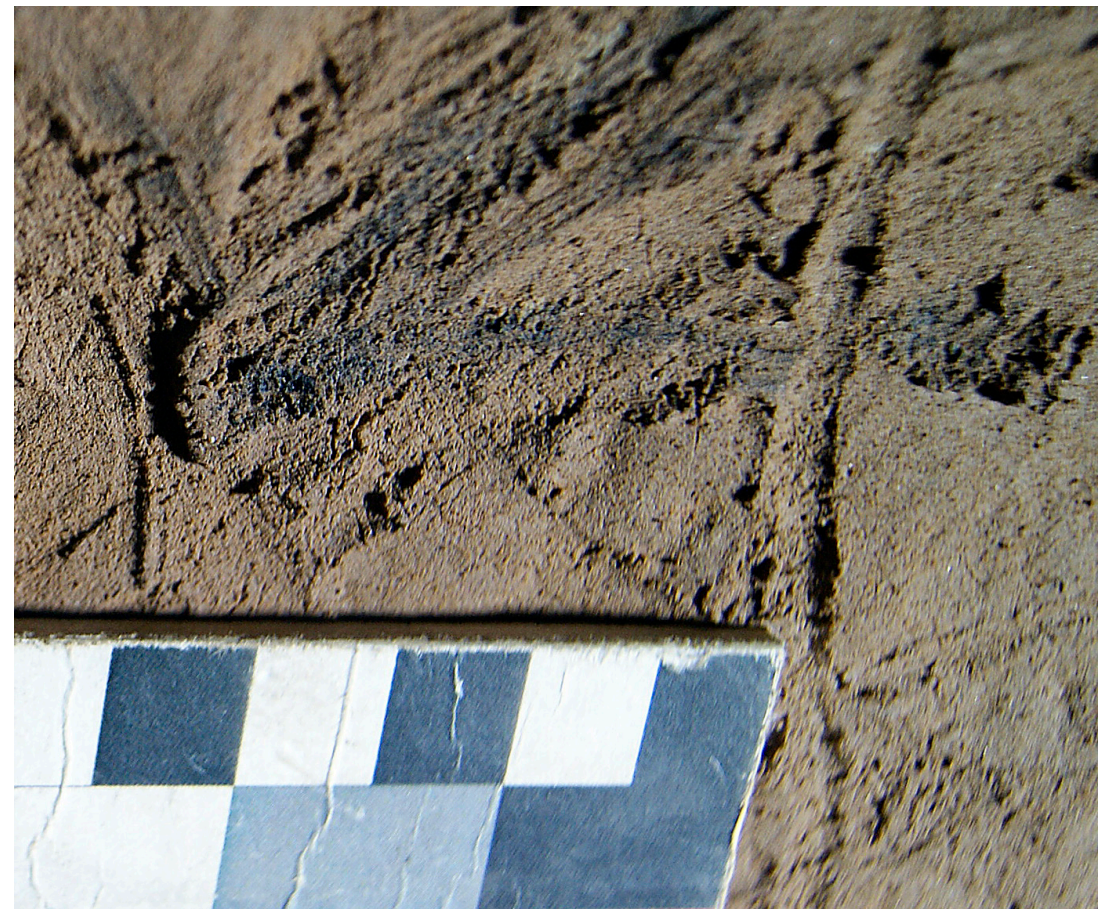

FIG. 4. Detalle de superposición de un trazo digital a uno de los trazos negros de carbón.

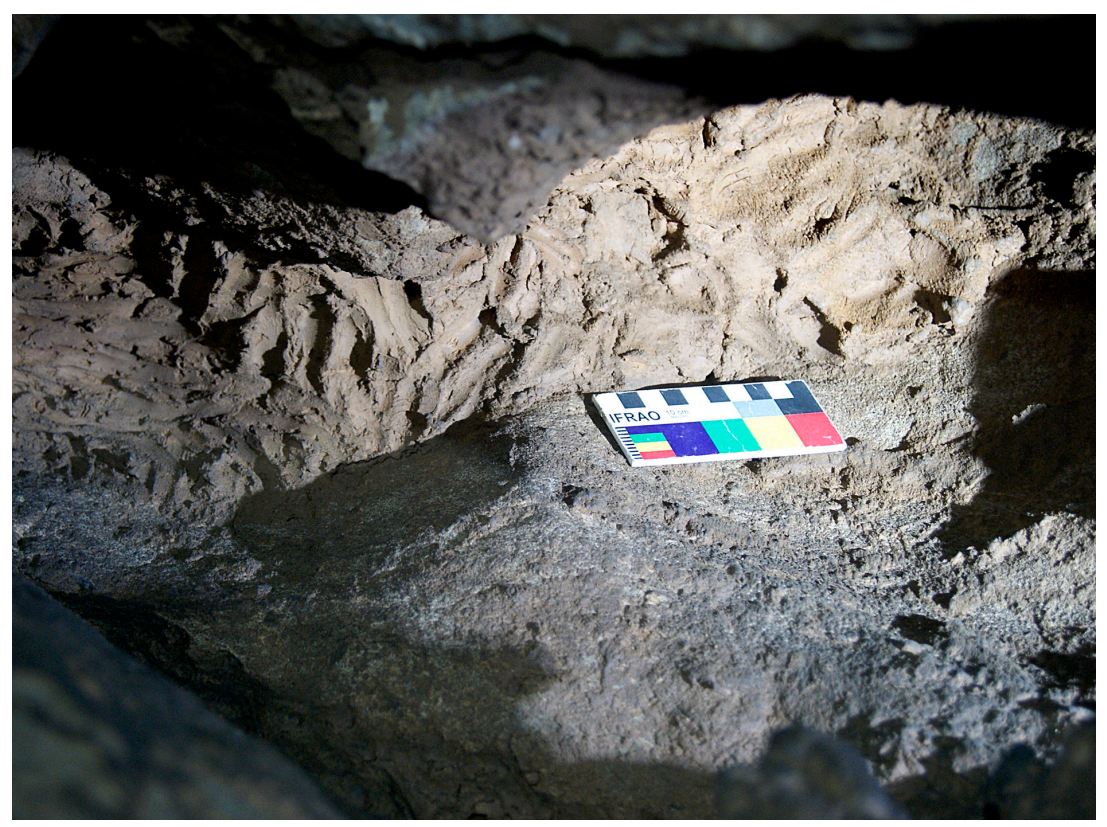

Fig. 5. Marcas que podrían estar relacionadas con la extracción de la arcilla en el extremo de la galería terminal.

2. Presencia de rebabas: casi todos ellos conservan rebabas, en algunas ocasiones de gran tamaño (Fig. 3). Estos productos de la sustracción de la arcilla sufren normalmente un proceso lento de desecación que los desprende de la pared y provoca finalmente su caída. Las de mayor tamaño son las primeras en desaparecer, por lo que la presencia de gran número de éstas no es habitual en trazos digitales de cronología paleolítica.

3. Ausencia de pátina y erosión: igual que los anteriores, no son elementos definitivos, pero sí suelen ser indicativos de una cierta antigüedad. En los grabados del Forcón no se aprecia la formación de pátinas -ni siquiera con lupa de $15 x-y$ en el interior de los surcos se pueden observar a menudo todavía las líneas de las huellas digitales, un elemento que normalmente se erosiona con relativa rapidez.

4. Superposiciones a trazos negros de aspecto reciente: en algunos casos, los trazos digitales aparecen superpuestos a algunas de las marcas negras que se han descrito (Fig. 4), por tanto, se puede deducir que, al menos, algunos de estos grabados son posteriores a ellas.

\section{Conclusión}

Con todo lo expuesto anteriormente, y a pesar de no poder descartarlo de forma categórica, no nos parece probable que las manifestaciones parietales de la Cueva del Forcón tengan un origen paleolítico. Ni 
siquiera creemos que haya argumentos suficientes para proponer que tengan un origen prehistórico. Juzgamos mucho más probable que fueran realizados en tiempos históricos, quizá contemporáneos a alguna de las dos inscripciones presentes en la misma galería y fechadas, respectivamente, en el s. XVII y la primera mitad del s. Xx.

Tampoco podemos afirmar que exista una finalidad simbólica en su elaboración. A modo de hipótesis, los vestigios podrían corresponderse con el resultado de la obtención de arcilla. A favor de esta propuesta, meramente especulativa, encontramos algunos rincones de la galería que han sido vaciados de arcilla y en los que hallamos este tipo de evidencias (Fig. 5). Pero con los datos disponibles, tanto la cronología exacta como la finalidad del dispositivo parietal de la Cueva del Forcón sigue siendo una incógnita. En consecuencia, y mientras no se documenten evidencias que demuestren lo contrario, juzgamos sensato que no se incluya este yacimiento en futuros inventarios de conjuntos con arte paleolítico.

\section{Bibliografía}

Baldellou, V. (1976): "La Prehistoria". En De URQuijo, A. (ed.): Alto Aragón, su historia, cultura $y$ arte. Madrid: Energía e Industrias Aragonesas, pp. 8-37.

Baldellou, V. (1985): "La Cueva del Forcón (La Fueva, Huesca)", Bolskan, 1, pp. 149-176.

Baldellou, V. (1987): "Avance al estudio de la Espluga de la Puyascada”, Bolskan, 4, pp. 3-42.

Baldellou, V. (1990): "La Prehistoria de Huesca: rasgos generales", Bolskan, 7, pp. 31-44.

Beltrán, A. (1990): "Arte rupestre prehistórico en Aragón”, Bolskan, 7, pp. 57-66.

Beltrán, A. (1997): "El Arte Rupestre Prehistórico", Caesarangusta, 72, pp. 311-347.

Casado, P. (1985): "Los grabados de la cueva de 'El Forcón”, Bolskan, 1, pp. 183-192.

Marco Simón, F. (1985): "Caballo y jabalíes en un bronce romano de la cueva del Forcón”, Bolskan,1, pp. 193-197.

Ruiz-Redondo, A. (2015): "Surcos en la arcilla: evaluación arqueológica de los hallazgos parietales de la Cueva de Coro Trasito (Tella, Huesca)". En Clemente, I.; Gassiot, E. y Rey, J. (eds.): Sobrarbe antes del Sobrarbe: pinceladas de Historia del Pirineo. Huesca: Centro de Estudios del Sobrarbe, pp. 33-42.

Sharpe, K. y Van Gelder, L. (2006): "The study of finger flutings", Cambridge Archaeological Journal, 16 (3), pp. 281-295.

Utrilla, P. (2000): El arte rupestre en Aragón. Zaragoza: Caja de Ahorros de la Inmaculada. 